\title{
Evolución del consumo de medicamentos de alto costo en Colombia
}

\author{
Jorge Enrique Machado Alba y Juan Carlos Moncada Escobar ${ }^{1}$
}

Forma de citar Machado Alba JE, Moncada Escobar JC. Evolución del consumo de medicamentos de alto costo en Colombia. Rev Panam Salud Publica. 2012;31(4):283-9.

RESUMEN Objetivo. Determinar el comportamiento del consumo de medicamentos de alto costo (MAC) durante 2005-2010 en una población de pacientes colombianos afiliados al Sistema General de Seguridad Social en Salud.

Métodos. Estudio descriptivo observacional; se analizaron datos de prescripción de fórmulas dispensadas desde 2005 a 2010 a todos los usuarios (1 674 517) de algún medicamento considerado de alto costo en 20 ciudades de Colombia. Se consideró la clasificación anatómica terapéutica y el número de pacientes, así como la facturación mensual por cada medicamento, la dosis diaria definida y el costo por 1000 habitantes/día.

Resultados. En todo el período de estudio, el valor facturado por MAC creció 847,4\%. Los antineoplásicos e inmunomoduladores constituyeron $46,3 \%$ del total facturado, antinfecciosos $15,2 \%$, preparaciones hormonales sistémicas 9,5\% y fármacos para el sistema nervioso 9,1\%. La mayoría de estos medicamentos fueron prescritos a las dosis diarias definidas recomendadas por la Organización Mundial de Salud, pero con altos costos por 1000 habitantes y día.

Conclusiones. En Colombia durante los últimos años se ha presentado una crisis debida al elevado gasto generado por los medicamentos más costosos. El crecimiento progresivo del gasto farmacéutico es mayor que el aumento de la cobertura del sistema sanitario del país. El sistema sanitario colombiano debe evaluar cuánto está dispuesto a pagar por los medicamentos más costosos para algunas morbilidades y qué estrategias debe implementar para sufragar estos gastos y así garantizar el acceso a los asegurados.

Palabras clave Control de costos; financiación de la salud; sustitución de medicamentos; utilización de medicamentos; economía farmacéutica; política de salud; Colombia.

El primer listado con 186 medicamentos esenciales fue publicado en 1977 por la Organización Mundial de la Salud (OMS). Desde entonces y hasta 2003 fue objeto de 11 revisiones y fue adoptado por más de 156 países —en la actualidad cuenta con 320 medicamentos en 559 formas de presentación. En general los criterios de selección de medicamentos esenciales han evolucionado hasta llegar al enfoque de medicina basada en la evidencia $(1,2)$.

\footnotetext{
1 Audifarma S.A., Departamento de Fármacoepidemiología, Pereira, Colombia. La correspondencia se debe dirigir a Jorge E Machado. Correo electrónico: machado@utp.edu.co
}

En este sentido los medicamentos esenciales, es decir los que satisfacen necesidades prioritarias para el cuidado de una población, son seleccionados teniendo en cuenta su relevancia en la salud pública, además de su eficacia, su seguridad y su costo-efectividad. De hecho, los listados nacionales de estos medicamentos varían según el perfil epidemiológico y el desarrollo económico de cada país (1). Los países pobres no los ofrecen todos, principalmente por razones de costo, lo cual crea una brecha entre los derechos en salud de sus habitantes y los de países con mejores condiciones económicas (3).
Los antineoplásicos e inmunomoduladores, antirreumáticos y antirretrovirales figuran entre los grupos excluidos de los listados de medicamentos esenciales y por lo tanto ofrecen mayores dificultades de acceso para quienes los necesitan (4-7). Se han encontrado asimismo grandes diferencias entre los costos de los medicamentos originales y los genéricos, por lo que algunos países han introducido políticas de acceso a estos últimos, aliviando así sus economías y ampliando las coberturas de atención en patologías de costoso tratamiento y que en el pasado fueron privilegio de países ricos, como ha ocurrido con el sida. Incluso

Errata: la afiliación del autor JE Machado Alba es Grupo de Investigación en Farmacoepidemiología y Farmacovigilancia, Universidad Tecnológica de Pereira, Pereira, Colombia. 
en algunos países se ofrecen programas de beneficios para facilitar el acceso a los medicamentos más costosos $(5,6$, 8-10). Un ejemplo es Colombia, donde hay una legislación que promueve el uso de medicamentos genéricos y obliga a su empleo dentro del sistema de salud, permitiendo un amplio desarrollo del mercado y una reducción significativa en los costos. En este país se definió un manual de medicamentos para el Plan Obligatorio de Salud (POS) que regula y actualiza periódicamente la lista de medicamentos a los cuales tiene acceso la población cubierta por los servicios de salud (10-14).

El listado de medicamentos esenciales de Colombia ofrece los mismos beneficios de racionalidad en el uso que cualquier otro, pero tiene las limitaciones propias de un formulario restrictivo para una población asegurada. Si bien se considera que esta medida ha reducido la brecha entre la cobertura y el acceso a los medicamentos, aún no ha resuelto de manera eficiente la situación de una proporción importante de la población aquejada por enfermedades que requieren medicamentos que no figuran en el listado y que se ve obligada a recurrir incluso a herramientas de orden legal para conseguirlos — situación similar a la planteada en otros países (11).

Sin embargo, cabe señalar que el Sistema General de Seguridad Social en Salud colombiano (SGSSS) dispone de una cuenta llamada "enfermedades catastróficas", para cuyo manejo el listado incluye un importante número de medicamentos. Por lo tanto para efectos de este trabajo, fueron considerados medicamentos de alto costo (MAC) aquellos que se encuentran por fuera del listado del plan obligatorio de salud y también aquellos que hacen parte de la cuenta de enfermedades catastróficas.

En años recientes se ha observado en el país un rápido crecimiento de las solicitudes de medicamentos no incluidos en el listado a través de herramientas contempladas por la ley, como los comités técnico-científicos (CTC) que están a cargo de los aseguradores o mediante fallos judiciales de tutela (12). El acelerado incremento de los medicamentos de alto costo que finalmente serán cubiertos por el Estado conlleva, además de los beneficios para la salud de las personas que los necesitan, una carga económica bastante pesada para el sistema sanitario del país.
Debido a la información limitada que existe sobre el perfil de utilización de los medicamentos de alto costo en Colombia, a su creciente consumo y a la carga financiera que traen aparejados, el presente estudio busca determinar el comportamiento de la utilización de medicamentos de alto costo en el período 2005-2010 en una población de pacientes afiliados al SGSSS. En la muestra se seleccionaron los medicamentos más representativos por frecuencia de uso y por costo, estableciéndose la dosis diaria definida (DDD) y el costo por 1000 habitantes/día para cada uno de los medicamentos que fueron objeto de análisis.

\section{MATERIALES Y MÉTODOS}

Se realizó un estudio descriptivo observacional de seguimiento sobre utilización de MAC a la cohorte de una población expuesta de 1674517 personas en Colombia afiliadas al régimen contributivo en una empresa promotora de salud (EPS). Esta muestra representó el $9 \%$ de la población asegurada en dicho régimen y 3,6\% de la afiliada al sistema sanitario del país. Se analizaron los datos de prescripción a partir de las fórmulas dispensadas durante el período 20052010 a todos los usuarios que recibieron algún medicamento considerado de alto costo en 20 ciudades de Colombia.

A partir de la base de datos de suministro de medicamentos que emplea la empresa que los dispensa (Audifarma S.A.), se tomaron los pacientes y las formulaciones objeto del trabajo. Se incluyeron los datos de los individuos de cualquier edad y sexo que hubiesen recibido algún MAC durante el período de 1 enero de 2005 a 31 diciembre de 2010 . Con esta información se identificaron los grupos de variables siguientes:

- Ciudad de residencia.

- Medicamentos dispensados según clasificación "anatómica, terapéutica, química (ATC, por sus siglas en inglés)": 1) antiinfecciosos de uso sistémico, 2) antineoplásicos y agentes inmunomoduladores, 3) antiparasitarios, insecticidas y repelentes, 4) dermatológicos, 5) músculo esquelético, 6) órganos de los sentidos, 7) preparaciones hormonales sistémicas, 8) sangre y órganos formadores de sangre, 9) sistema cardiovascular, 10) sistema genitourinario y hormonas sexuales, 11) sistema nervioso, 12) sistema res- piratorio y 13) tracto alimentario y metabolismo.

- Número de pacientes y facturación mensual por cada medicamento.

- Cuenta de alto costo por la cual fue dispensado el MAC: 1) catastróficas, 2) CTC y 3) tutelas.

- Costos globales de los medicamentos dispensados. Se calculó el costo absoluto para cada año analizado de todos los principios activos y también el costo por 1000 habitantes/día (CHD) $(\mathrm{CHD}=[$ costo $/ 365 \times$ No. habitantes $] \times$ 1000). En el caso de las asociaciones antimicrobianas no se disgregó el costo por principio activo.

Para cuantificar la dispensación se utilizó como unidad técnica de medida la dosis diaria definida recomendada por la OMS como estándar internacional para la realización de estudios fármacoepidemiológicos, expresándose en forma de dosis por habitante por día (DHD).

Los valores facturados por cada mes de 2005 a 2010, fueron convertidos a $\mathrm{su}$ equivalente monetario a diciembre de 2010, con base en la variación general del índice de precios al consumidor publicado por el Departamento Administrativo Nacional de Estadísticas en su página web. Para la conversión de la inversión mensual y anual a dólares de Estados Unidos, se utilizó la información histórica de la tasa representativa de mercado, al último día hábil de cada mes y año publicada por el Banco de la República en su página web.

Se determinó la evolución de los 14 medicamentos más representativos para el alto costo en cuanto a frecuencia de uso y facturación mensual. El protocolo fue sometido a revisión científico-técnica y bioética por las instancias correspondientes de la Facultad de Ciencias de la Salud de la Universidad Tecnológica de Pereira y del asegurador participante, en la categoría de investigación sin riesgo. La base de datos fue revisada por el departamento de fármaco-epidemiología de la empresa encargada de la dispensación farmacéutica.

\section{RESULTADOS}

La población del estudio creció progresivamente desde 1068077 usuarios en 2005 hasta 1674517 en 2010 (56,7\%), aunque durante el mismo período el número de pacientes que accedieron a MAC creció $1166,5 \%$ y el valor factu- 
rado $847,4 \%$ (figura 1 ). El promedio de facturación por paciente, sin embargo, mostró una tendencia decreciente (figura 2). El número de pacientes que accedió a los MAC incluidos en el POS a través de la subcuenta "catastróficas" reveló un comportamiento estable, sin mayores cambios, mientras que aquellos que lo hicieron a través del instrumento legal "tutela" tuvieron su máximo pico en julio de 2008, después del cual comenzaron a descender. La variación más significativa la mostraron los pacientes que accedieron a través de los CTC, los cuales en 6 años crecieron 10 103\% (figura 3).

Al diferenciar la evolución de la facturación por subcuenta de alto costo se pudo observar que no hubo crecimiento para los medicamentos que se encontraban incluidos en el POS, mientras que el valor se multiplicó para los obtenidos a través de tutelas y subió $2619 \%$ para los de CTC — casi duplicándose año tras año (figura 4).

De acuerdo a la clasificación ATC, se encontró que el grupo de medicamentos más representativo por el costo que generó fue el de agentes antineoplásicos e inmunomoduladores, los cuales durante los 6 años promediaron 46,3\% del valor facturado por todos los MAC, seguido de los antiinfecciosos de uso sistémico $(15,2 \%)$, las preparaciones hormonales sistémicas $(9,5 \%)$ y los fármacos para sistema nervioso $(9,1 \%)$, en tanto que los nueve grupos restantes solo generaron $19,8 \%$ de la facturación total. El grupo de antineoplásicos e inmunomoduladores mantuvo el mismo porcentaje de facturación a lo largo de estos años, mientras que los psicofármacos mostraron un crecimiento cercano a $61,1 \%$ y los antiinfecciosos disminuyeron alrededor de $76,8 \%$.

La mayoría de los medicamentos evaluados estaban siendo manejados a dosis medias que se encontraban en el rango recomendado por la literatura y muy cercanos a la DDD sugerida por la OMS. Por ejemplo para adalimumab, cuya DDD es $2,9 \mathrm{mg}$, los pacientes recibieron en 2010 una media de 0,98 DDD, para etanercept (DDD $7 \mathrm{mg}$ ) 1,09 DDD, para la asociación lopinavir/ritonavir (DDD 0,8 g) 1,04 DDD, para abacavir (DDD 0,6 g) 1,13 DDD, para micofenolato de mofetilo (DDD 2 g) 0,78 DDD, para interferón beta $1 \mathrm{~A}$ de $6 \mathrm{MUI}$ (DDD 4,3 mg) 1,0 DDD y para lamotrigina (DDD 0,3 g) 1,1 DDD. En medicamentos como

FIGURA 1. Evolución del número de pacientes y de la facturación mensual de MAC registrada en usuarios de una EPS. Colombia, 20 ciudades, 2005-2010 (en miles de dólares estadounidenses ${ }^{\mathrm{a}}$ )

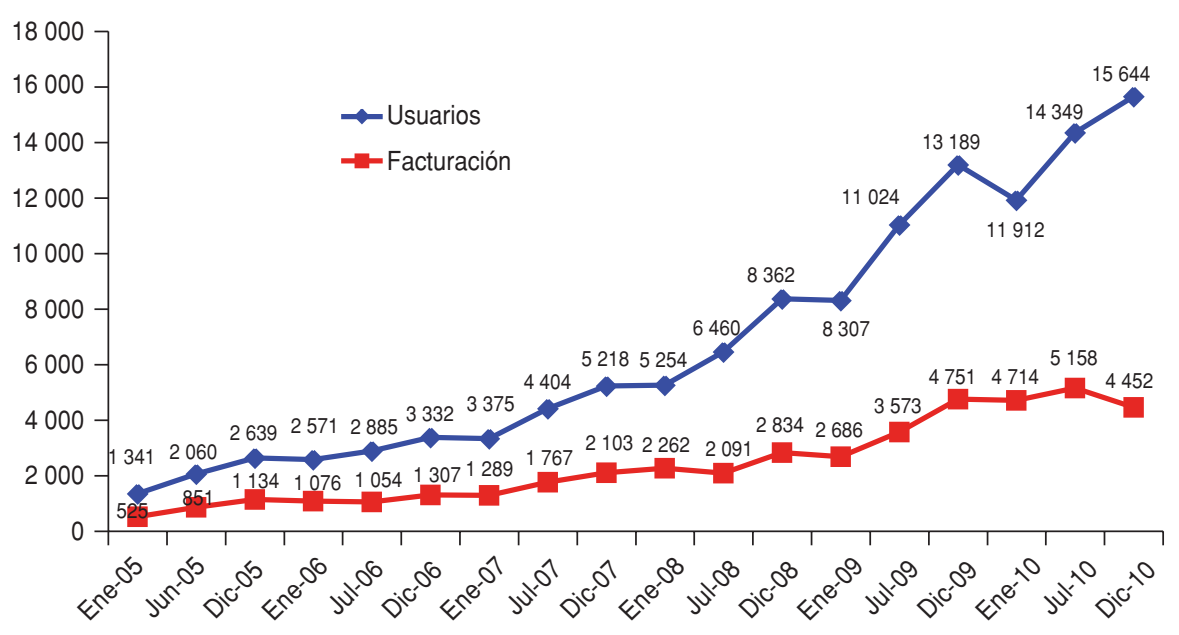

Fuente: Elaboración de los autores con datos del Departamento de Farmacoepidemiología-Audifarma S.A., Pereira, Colombia. Nota: MAC: medicamentos de alto costo; EPS: empresa promotora de salud.

a Ajustados según la tasa representativa del mercado emitida por el Banco de la República, Colombia.

FIGURA 2. Evolución promedio facturado por MAC por paciente en una EPS. Colombia, 20 ciudades, 2005-2010 (en dólares estadounidenses ${ }^{\mathrm{a}}$ )

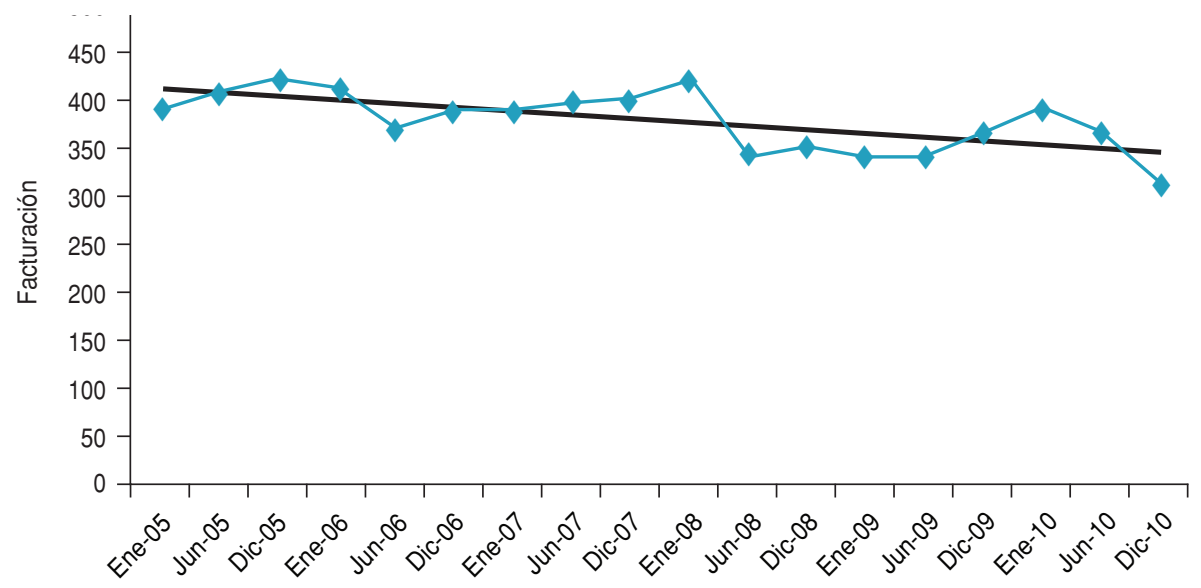

Fuente: elaboración de los autores con datos del Departamento de Farmacoepidemiología-Audifarma S.A., Pereira, Colombia. Nota: MAC: medicamentos de alto costo; EPS: empresa promotora de salud.

${ }^{a}$ Ajustados según la tasa representativa del mercado emitida por el Banco de la República, Colombia.

la somatropina, cuya DDD es $2 \mathrm{U}$, se encontró que en promedio recibieron el doble de la DDD —aproximadamente de 2,14 DDD. Los datos son similares para todos los años del estudio.

En el cuadro 1 se presenta la evolución del consumo de los medicamentos analizados. Como se puede ver, entre 2005 y 2010 rituximab registró un incremento de $3028,2 \%$, imatinib $1464,8 \%$, adalimumab $768,8 \%$, etanercept $50 \mathrm{mg} \mathrm{588 \%}$, micofenolato de mofetilo
$315,2 \%$, infliximab $314,4 \%$, lamotrigina $258,5 \%$, somatropina $242,3 \%$, interferón beta 1 b de 8 MUI 206,4\% y abacavir 198,6\%. El antiinhibidor factor VIII-IX creció 778,9\% en solo 3 años, mientras que otros fármacos tuvieron crecimientos menos marcados, como el interferón beta $1 \mathrm{~A}$ de $6 \mathrm{MUI}$, que aumentó apenas $88,8 \%$. Otros medicamentos mantuvieron un consumo estable (interferón beta 1A de 12MUI) o incluso menor, como fue el caso de lopinavir/ritonavir, cuyo con- 
FIGURA 3. Evolución del número de pacientes en cuenta de alto costo de una EPS, según accedieran mediante la subcuenta "Catastróficas", el instrumento "Tutela" o los CTC. Colombia, 20 ciudades, 2005-2010

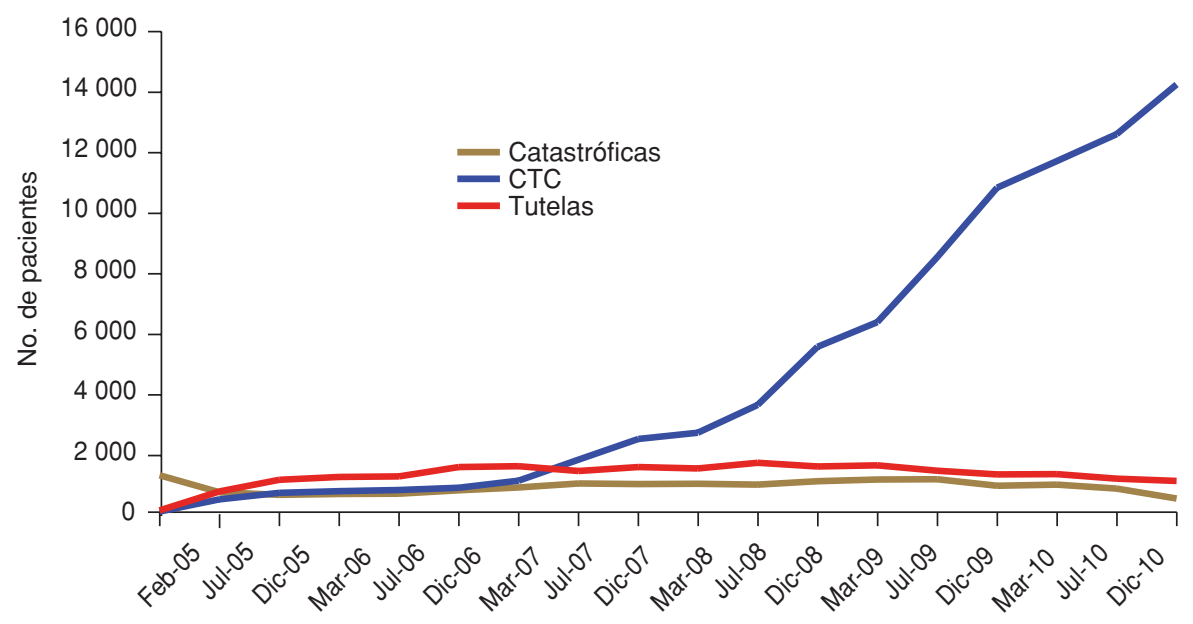

Fuente: elaboración de los autores con datos del Departamento de Farmacoepidemiología-Audifarma S.A., Pereira, Colombia. Nota: EPS: empresa promotora de salud; CTC: comités técnico-científicos.

FIGURA 4. Facturación anual en cuenta de alto costo de usuarios de una EPS, según accedieran mediante la subcuenta "Catastróficas", el instrumento "Tutela" o los CTC. Colombia, 20 ciudades, 2005-2010 (en dólares estadounidenses) ${ }^{\mathrm{a}}$



Fuente: elaboración de los autores con datos del Departamento de Farmacoepidemiología-Audifarma S.A., Pereira, Colombia. Nota: EPS: empresa promotora de salud; CTC: comités técnico-científicos.

a Ajustados según la tasa representativa del mercado emitida por el Banco de la República, Colombia

sumo se redujo $88,4 \%$. La presentación de etanercept de $25 \mathrm{mg}$ prácticamente entró en desuso.

En el cuadro 1 también se muestra la evolución de los costos de MAC. Como se puede ver, en el último año analizado el asegurador tuvo que invertir US\$ 5,01 por cada 1000 habitantes para suministrar adalimumab a los pacientes con artritis reumatoide, es decir 8,7 veces más que lo que gastaba en 2005, situación similar a la que se dio con otros fármacos como etanercept $50 \mathrm{mg}$, imatinib, rituximab, interferones, micofenolato de mofetilo y lamotrigina. En el caso de los antirretrovirales lopinavir/ritonavir y abacavir, se halló que el CHD no creció en relación con la DHD, sino que contrariamente registraron una merma en la facturación.

\section{DISCUSIÓN}

El gasto creciente que trae aparejada la mayor utilización de medicamentos representa un gran reto para la mayoría de los sistemas de salud. En Colombia durante los últimos años se ha presentado una crisis debida al elevado gasto sanitario, en especial el generado precisamente por los medicamentos más costosos. En este trabajo se pudo mostrar el crecimiento progresivo del gasto farmacéutico, que rebasa al aumento de la cobertura del sistema, el cual entre 2005 y 2010 no alcanzó a 13\% para el régimen contributivo (15).

Los medicamentos que generaron gastos más altos al sistema fueron los antineoplásicos e inmunomoduladores, como ha sido reportado por otros autores, sobre todo los empleados para el tratamiento de leucemias y artritis reumatoide, aunque también los utilizados para tratar el $\mathrm{VIH} /$ sida, los trasplantes, la esclerosis múltiple y los trastornos convulsivos (16). En particular aumentó el gasto en medicamentos que pertenecen al grupo desarrollado mediante biotecnología, y entre ellos de manera especial los anticuerpos monoclonales, tal como ha ocurrido en todo el mundo (17).

Algunos sistemas han incorporado políticas que restringen el número de fármacos cuyo valor le es reembolsado a los pacientes, incluyendo el cobro de copagos fijos por fármaco, o los pagos de un valor parcial o total del medicamento cuando otro que está incluido en el aseguramiento está disponible y es de menor valor, e incluso niveles de copago diferencial si se trata de medicamentos genéricos o de marca $(18,19)$. En Inglaterra e Irlanda, por ejemplo, se han venido restringiendo los presupuestos para medicamentos, lo que ha limitado el volumen de prescripciones, incrementando el uso de genéricos (20).

Varios estudios muestran que la introducción - o el incremento- de los copagos ha disminuido el uso de medicamentos y en consecuencia el gasto farmacéutico, debido a que provocó que los pacientes respondieran descontinuando los fármacos innecesarios. El problema es que los investigadores también observaron reducciones en medicamentos vitales o necesarios para tratar condiciones crónicas, lo cual potencialmente podría plantear riesgos para la salud (18). Incluso la misma investigación reportó un incremento de la atención sanitaria 
CUADRO 1. Evolución del consumo de los 14 MAC más significativos por DDD y por CHD en una EPS. Colombia, 20 ciudades, 2005-2010

\begin{tabular}{|c|c|c|c|c|c|c|c|c|c|c|c|c|}
\hline \multirow[b]{2}{*}{ Medicamento } & \multicolumn{6}{|c|}{ DDD } & \multicolumn{6}{|c|}{$\mathrm{CHD}^{\mathrm{a}}$} \\
\hline & 2005 & 2006 & 2007 & 2008 & 2009 & 2010 & 2005 & 2006 & 2007 & 2008 & 2009 & 2010 \\
\hline Abacavir 300 mg & 9,08 & 20,76 & 28,12 & 42,92 & 32,79 & 18,03 & 0,30 & 0,54 & 0,81 & 1,19 & 0,50 & 0,11 \\
\hline Adalimumab $40 \mathrm{mg}$ & 0,02 & 0,05 & 0,07 & 0,11 & 0,14 & 0,17 & 0,57 & 1,36 & 2,08 & 3,19 & 4,73 & 5,01 \\
\hline Antinhibidor factor VIII-IX 500 UI & 0 & 0 & 0 & 1,68 & 3,32 & 13,08 & 0,00 & 0,00 & 0,00 & 0,51 & 0,87 & 3,86 \\
\hline Etanercept 50 mg & 0 & 0 & 0,009 & 0,018 & 0,062 & 0,070 & 0,00 & 0,00 & 0,28 & 0,50 & 1,87 & 1,91 \\
\hline Imatinib 400 mg & 0,003 & 0,006 & 0,005 & 0,005 & 0,005 & 0,044 & 1,25 & 2,13 & 2,53 & 2,64 & 2,22 & 2,20 \\
\hline Infliximab 100 mg & 0,02 & 0,03 & 0,06 & 0,06 & 0,08 & 0,06 & 0,57 & 1,11 & 2,21 & 2,57 & 3,49 & 2,71 \\
\hline Interferon beta 1-A 12MUI & 0,06 & 0,06 & 0,05 & 0,07 & 0,06 & 0,05 & 0,42 & 0,42 & 0,47 & 0,69 & 0,61 & 0,54 \\
\hline Interferon beta 1-A 6MUI & 0,06 & 0,06 & 0,06 & 0,07 & 0,07 & 0,05 & 0,71 & 0,82 & 1,09 & 1,22 & 1,25 & 1,14 \\
\hline Interferon beta 1B 8MUI & 0,14 & 0,22 & 0,29 & 0,33 & 0,38 & 0,28 & 0,75 & 1,25 & 1,90 & 2,28 & 2,56 & 2,11 \\
\hline Lamotrigina 100 mg & 23,65 & 30,06 & 41,63 & 50,41 & 57,58 & 61,14 & 0,23 & 0,24 & 0,37 & 0,47 & 0,53 & 0,63 \\
\hline Lopinavir+Ritonavir 200+50 mg & 51,73 & 8,35 & 40,73 & 53,86 & 47,39 & 33,34 & 1,37 & 0,26 & 1,14 & 1,45 & 1,07 & 0,37 \\
\hline Micofenolato Mofetilo $500 \mathrm{mg}$ & 3,94 & 4,95 & 6,61 & 8,93 & 9,96 & 12,43 & 0,44 & 0,57 & 0,89 & 1,26 & 1,40 & 1,37 \\
\hline Rituximab 500 mg & 0,000003 & 0,00011 & 0,00003 & 0,00007 & 0,00008 & 0,00010 & 0,14 & 0,70 & 1,20 & 2,65 & 3,47 & 2,39 \\
\hline Somatropina $16 \mathrm{UI}$ & 0,51 & 0,82 & 1,15 & 1,20 & 1,18 & 1,24 & 1,88 & 2,10 & 2,61 & 2,65 & 2,63 & 3,16 \\
\hline
\end{tabular}

Fuente: elaboración de los autores con datos del Departamento de Farmacoepidemiología-Audifarma S.A, Pereira, Colombia.

Nota: MAC: medicamentos de alto costo; DDD: dosis diarias definidas [por 100000 habitantes]; CHD: costo por [1 000 habitantes] por día; EPS: empresa promotora de salud.

a En dólares estadounidenses, ajustados según la tasa representativa del mercado emitida por el Banco de la República, Colombia.

cuando la medida fue tomada en población vulnerable. Finalmente concluyeron que el copago directo de fármacos puede no causar daños importantes, pero siempre y cuando no se aplique a medicamentos esenciales (18).

En China, cuyas reformas económicas han mejorado el acceso a tecnologías médicas y nuevos tratamientos, también se ha elevado el costo de la atención sanitaria asociado al alto costo de nuevos medicamentos, los reembolsos ocasionados y la sobre utilización de fármacos. Con objeto de reducir dicho gasto, se están planteando medidas para controlar el precio de los nuevos fármacos y penalizar a los agentes causantes de los altos costos - propuesta similar a la recientemente planteada en Colombia (21). Israel ha implementado asimismo medidas de regulación de precios de los medicamentos (22).

La mayoría de países europeos regulan los precios de los productos farmacéuticos, incluyendo medidas que emplean precios internacionales de referencia (de los países que tienen bajos precios), sistemas de información de precios internos que promuevan su competitividad en el mercado interno y listas positivas de reembolso a los pacientes que consumen genéricos. No obstante estas medidas, todavía no han identificado la solución para controlar los gastos en medicamentos (23). En Portugal por ejemplo, las políticas adoptadas para controlar el gasto farmacéutico no han sido exitosas. Una de ellas que invitaba a incrementar la competencia en el mercado de medicamentos y la introducción de precios de referencia, tuvo un efecto transitorio de menos de un año, con un nueva y rápida subida en los precios que transfirió finalmente el costo a los pacientes sin que afectara las ventas finales (19).

En general, también han crecido sostenidamente los gastos en medicamentos de uso hospitalario, ocasionados principalmente por los llamados medicamentos de alto costo -entre los cuales los oncológicos son la gran mayoría. Ante este desafío, es perentorio que los sistemas de salud inviertan más recursos presupuestarios dirigidos a la prevención de enfermedades crónicas generadoras de un elevado gasto (16). En el caso de Colombia, hay que señalar que el gasto en MAC se redujo o estabilizó a lo largo de 2010, luego que en 2009 el Ministerio de Protección Social declaró una "emergencia social" e introdujo normas que restringieron el acceso a los servicios de salud y a algunos de los MAC. El propósito central fue controlar la sobre facturación de medicamentos, el recobro a veces ilegal de algunos aseguradores y el abuso de prescriptores y pacientes que fomentaban las tutelas sin previo análisis por CTC (24). Otro agravante es que los médicos ignoran los costos, lo cual combinado con su tendencia a subestimar el precio de medicamentos caros y a sobreestimar el precio de los baratos no consideran la variable costo al momento de prescribir (25). Esta discrepancia podría tener profundas implicaciones en el gasto farmacéutico, de tal forma que se requiere enfocar más la atención en brindar educación continuada a los médicos acerca de los costos y el acceso a la información sobre precios para que tomen conciencia de su importancia y lo reflejen prescribiendo el fármaco que pueda resultar más costo-efectivo (25).

Vale mencionar también que, en el caso colombiano, había un gran número de pacientes que no tenían acceso a una terapia adecuada y el SGSSS, mediante los CTC, abrió la puerta para ofrecerles acceso a medicamentos no incluidos en el listado oficial, provocando lógicamente un acelerado crecimiento en el número de pacientes consumidores de MAC. Por fortuna, algunas de las medidas tomadas permitieron mantener e incluso reducir el gasto en medicamentos por individuo en los últimos años. Dado que se descuenta que no debe ni puede haber restricciones para acceder al mejor tratamiento disponible, los esfuerzos para recortar gastos deben enfocarse en prevenir el uso poco racional, establecer precios de referencia y promover la prescripción de genéricos. Dado que el libre mercado por definición no controla los precios de los fármacos, es preciso implementar otros mecanismos de control y monitoreo, ya que incluso se ha demostrado que los márgenes de beneficio de los productos genéricos suelen ser mayores que los de los productos innovadores (26).

Se debe agregar que los datos analizados en el presente estudio corresponden 
a uno solo de los aseguradores del país, por lo cual, aun cuando es uno de los cinco con mayor número de pacientes, no necesariamente debe reflejar la realidad de toda la población colombiana afiliada. Al margen de tal limitación, el trabajo logró describir la tendencia en la evolución del comportamiento de los medicamentos generadores de mayor gasto para un asegurador en salud y para el sistema sanitario nacional, hallándose que los inmunomoduladores y los anteneoplásicos son los principales responsables.

Pese a las dudas sobre la efectividad de las medidas de contención del gasto en medicamentos, países como Colom- bia - con claras restricciones en sus presupuestos sanitarios- deben estudiar y adoptar estrategias efectivas que limiten el crecimiento desmedido que se viene registrando en el costo de los medicamentos. También importa preguntarse cuánta responsabilidad tiene la industria farmacéutica al mantener costos elevados en medicamentos que son imprescindibles para tratar de aliviar muchos de los problemas que afectan a la salud de la población y qué implicaciones tienen sus intereses comerciales (27).

Más allá de los costos simples que están generando los medicamentos al sistema sanitario colombiano, se debe evaluar cuánto está dispuesto a pagar el país por las morbilidades más costosas y qué estrategias se deben implementar para sufragar dichos gastos y así garantizar el acceso a los asegurados. Por último, se recomienda efectuar nuevas evaluaciones de los resultados logrados con las medidas llevadas a cabo por el Estado en relación con el gasto farmacéutico y su impacto económico, social $\mathrm{y}$, particularmente, en la salud de los colombianos.

Agradecimiento. Los autores agradecen a Diego Fernando Díaz su valioso apoyo en los cálculos cambiarios requeridos para el estudio.

\section{REFERENCIAS}

1. Laing R, Waning B, Gray A, Ford N, 't Hoen E. 25 years of the WHO essential medicines lists: progress and challenges. Lancet. 2003;361:1723-9.

2. Seuba $X$. A human rights approach to the WHO Model List of Essential Medicines. Bull World Health Organ. 2006;84(5):405-7.

3. Ashcroft RE. Access to essential medicines: a Hobbesian social contract approach. Dev World Bioeth. 2005;5(2):121-41.

4. Drummond M, Mason A. European perspective on the costs and cost-effectiveness of cancer therapies. Journal of Clinical Oncology. 2007;25(2):191-5.

5. Lu CY, Williams KM, Day RO. Access to tumour necrosis factor inhibitors for rheumatoid arthritis treatment under the Australian Pharmaceutical Benefits Scheme: are we on target? Intern Med J. 2006;36(1):19-27.

6. Bartlett JA, Muro EP. Generic and Branded Drugs for the Treatment of People Living with HIV / AIDS. J Int Assoc Physicians AIDS Care (Chic). 2007;6(1):15-23.

7. Campos-Olazabal P. Risks and benefits of the new antiepileptic drugs. Rev Neurol. 2006; 42(3):57-60.

8. Rojo P. El acceso a los medicamentos esenciales en los países pobres. Gac Sanit. 2001; 15(6):540-5.

9. Faunce TA. The UNESCO Bioethics Declaration 'social responsibility' principle and costeffectiveness price evaluations for essential medicines. Monash Bioeth Rev. 2005;24(3): 10-9.

10. Garenne M, Candau D, Guimier JM, Badiane M, Diop AC, Teuliéres LC. Access to medicines in Senegal: results of a sample survey. Trop Doct. 2006;36(1):5-8.

11. Hogerzeil HV, Samson M, Vidal Casanovas J, Rahmani-Ocora L. Is access to essential medicines as part of the fulfillment of the right to health enforceable through the courts? Lancet. 2006;368:305-11.

12. Ministerio de Salud de Colombia.Consejo Nacional de Seguridad Social en Salud. Acuerdo 228 de 2002. Disponible en: http:/ / www.col.ops-oms.org/medicamentos / Documentos/ac228_02.htm Acceso el 21 de noviembre de 2011.

13. Ministerio de la Protección Social de Colombia. Consejo Nacional de Seguridad Social en Salud. Acuerdo 282 de 2004. Disponible en: http:/ / www.cntv.org.co/cntv_bop/basedoc/ acuerdo/cnsss/acuerdo_cnsss_0282_2004. html Acceso el 9 de enero de 2012.

14. Ministerio de la Protección Social de Colombia. Consejo Nacional de Seguridad Social en Salud. Acuerdo 0336 de 2006. Disponible en: http:/ / www.pos.gov.co/Documents / Archivos/Normatividad_Regimen_Contrib utivo/acuerdo_336_2006.pdf Acceso el 21 de noviembre de 2011.

15. Ministerio de la Protección Social de Colombia. Estadísticas: Base de datos única de afiliados, consolidado 2010. Disponible en: http: / / www.minproteccionsocial.gov. co/estadisticas/default.aspx Acceso el 21 de noviembre de 2011.

16. Niezen MG, Stolk EA, Steenhoek A, Uyl-De Groot CA. Inequalities in oncology care: Economic consequences of high cost drugs. Eur J Cancer. 2006;42(17):2887-92.

17. Cohen J, Wilson A. New challenges to medicare beneficiary access to mAbs. MAbs. 2009; 1(1):56-66.

18. Austvoll-Dahlgren A, Aaserud M, Vist G, Ramsay C, Oxman AD, Sturm H, et al. Pharmaceutical policies: effects of cap and copayment on rational drug use. Cochrane Database Syst Rev. 2008;23(1):CD007017.

19. Barros PP, Nunes LC. The impact of pharmaceutical policy measures: anendogenous structural-break approach. Soc Sci Med. 2010; 71(3):440-50.

20. Sturm H, Austvoll-Dahlgren A, Aaserud M, Oxman AD, Ramsay C, Vernby A, et al. Pharmaceutical policies: effects of financial incentives for prescribers. Cochrane Database Syst Rev. 2007;18(3):CD006731.

21. Jufang S, Huiyun S. Some views about the high cost of medical care in China. Southeast Asian J Trop Med Public Health. 2010;41(1):240-2.

22. Sax P, Shmueli A. Impact of pharmaceutical regulation and policies on health system performance goals in Israel. Adv Health Econ Health Serv Res. 2010;22:77-101.

23. Barros PP. Pharmaceutical policies in European countries. Adv Health Econ Health Serv Res. 2010;22:3-27.

24. Presidencia de la República de Colombia. Decreto 4975 de 2009. Disponible en: http:/ / www.elabedul.net/Documentos / Leyes/2009/Decreto_4975.pdf Acceso el 21 de noviembre de 2011.

25. Allan GM, Lexchin J, Wiebe N. Physician awareness of drug cost: a systematic review. PLoS Med. 2007;4(9):1486-96.

26. Babar ZU, Ibrahim MI, Singh H, Bukahri NI, Creese A. Evaluating drug prices, availability, affordability, and price components: implications for access to drugs in Malaysia. PLoS Med. 2007;4:466-75.

27. Velásquez G. El acceso global a los medicamentos en el contexto internacional actual. Biomédica. 2011;31(2):161-3.

Manuscrito recibido el 2 de mayo de 2011. Aceptado para publicación, tras revisión, el 14 de noviembre de 2011. 
ABSTRACT Objective. Determine the patterns of consumption of high-cost drugs (HCD) during the 2005-2010 period in a population of Colombian patients enrolled in the General System of Social Security in Health.

\section{Evolution of consumption of high-cost drugs in Colombia}

Methods. An observational descriptive study was conducted. The prescription data of formulas of any drug considered to be high-cost dispensed to all users (1 674 517) in 20 cities of Colombia between 2005 and 2010 were analyzed. The anatomical therapeutic classification was considered, and the number of patients as well as monthly invoicing for each drug, the daily dose defined, and the cost per 1000 inhabitants/ day were defined.

Results. Over the entire study period, the amount invoiced for HCDs increased by $847.4 \%$. Antineoplastic and immunomodulator drugs accounted for $46.3 \%$ of the total invoicing. The other drugs were anti-infectives (15.2\%), systemic hormonal preparations $(9.5 \%)$, and drugs for the nervous system (9.1\%). Most of these drugs were prescribed at the daily doses defined as recommended by the World Health Organization, but with high costs per 1000 inhabitants/day.

Conclusions. In Colombia a crisis has occurred in recent years due to the high spending generated by the most expensive drugs. The progressive growth of pharmaceutical spending is greater than the increased coverage by the country's health system. The Colombian health system should evaluate how much it is willing to pay for the most expensive drugs for some diseases and what strategies should be implemented to cover these expenses and thus guarantee access to the insured.

Key words Cost control; financing, health; drug substitution; drug utilization; economics, pharmaceutical; health policy; Colombia. 